\title{
Excitation Fields in a Superconducting Global String
}

\author{
J.R. Morris* \\ Department of Chemistry/Physics/Astronomy, \\ Indiana University Northwest, 3400 Broadway, \\ Gary, Indiana 46408
}

PACS : 11.2\%.+d, 11.30.Qc, 98.80.Cq

\begin{abstract}
A model of a straight superconducting global cosmic string is examined in a setting wherein the string supports a charge/current pulse described by a travelling wave along the string. Linearized field equations are obtained for fluctuations of the scalar and vector fields of the theory, and a set of approximate particular solutions are found for the case in which the linear charge density and the current of the string have equal magnitudes. Although the equations of motion seem to suggest that the scalar and vector excitation fields are massive inside the string core, the particular solutions show that they behave as effectively massless fields which propagate at the speed of light along the string along with the primary charge/current pulse. The effect of the mass parameter is to modulate the radial profile of the excitation fields. The vector excitation field generates radial and angular components for both the electric and magnetic fields, but the particular solutions do not describe the emission or absorption of electromagnetic radiation from the string.
\end{abstract}

*E-mail address: jmorris@iunhaw1.iun.indiana.edu 


\section{INTRODUCTION}

A superconducting global string can arise from a situation wherein, by the Witten mechanism [1], the spontaneous symmetry breaking of a global $U(1)$ symmetry is accommodated by the formation of a scalar condensate which breaks a gauged $U(1)$ symmetry in the core of the string. The string can thus be endowed with a charge per unit length $Q$ and a current $I$ which are determined by the complex scalar field which forms the condensate and the vector gauge field which is coupled to it. $Q$ and $I$ may be either time independent or time-dependent, and in particular $Q$ and $I$ can be described in terms of travelling waves along the string [2]. A basic ansatz [3] for a straight superconducting global string can be constructed where the moduli of the scalar fields and the vector gauge field structure function depend only upon the radial distance $r$ from the center of the string and the phase of the scalar condensate field depends upon the position $z$ along the string, along with the time $t$.

Here, attention is focused upon a situation where relatively small fluctuations of the fields about their basic ansatz values are considered. These small fluctuations, or excitation fields, are treated as classical fields, and linearized equations for the excitation fields are obtained. By implementing an ansatz, some approximate particular solutions of the linear excitation field equations can be extracted. In particular, approximate solutions describing the excitation fields are found for the specific case where a charge/current pulse travels along the string with $Q^{2}-I^{2}=0$. Although the equations of motion seem to imply that the scalar and vector excitation fields are massive inside the string core, the particular solutions that are found show that they are effectively massless fields that propagate at the speed of light along the string with the charge/current pulse. The excitation fields give rise to additional charge and current densities, along with electromagnetic fields. The electric and magnetic fields are each found to possess both a radial and an angular component, but there is no absorption or emission of electromagnetic radiation from the string.

The model describing the superconducting global string is presented in section II. The excitation field equations are described in section III, and approximate solutions are obtained in section IV. A summary and discussion forms section V.

\section{THE SUPERCONDUCTING GLOBAL STRING}

\section{A. General Field Equations}

Consider a straight, infinite global cosmic string [4], [5] lying along the $z$ axis

(we use cylindrical coordinates $(r, \theta, z)$ ), with a complex-valued scalar string field $\chi$ 
interacting with a complex-valued scalar field $\phi$ coupled to a $U(1)$ gauge field $A_{\mu}$, as described by the Lagrangian

$$
L=\partial^{\mu} \chi^{*} \partial_{\mu} \chi+\left(D^{\mu} \phi\right)^{*}\left(D_{\mu} \phi\right)-V-\frac{1}{4} F^{\mu \nu} F_{\mu \nu}
$$

where

$$
D_{\mu}=\nabla_{\mu}+i e A_{\mu}, \quad F_{\mu \nu}=\partial_{\mu} A_{\nu}-\partial_{\nu} A_{\mu},
$$

with the potential $V$ given by

$$
V=\lambda\left(\chi^{*} \chi-\frac{1}{2} \eta^{2}\right)^{2}+2 f\left(\chi^{*} \chi-\frac{1}{2} \eta^{2}\right) \phi^{*} \phi+m^{2} \phi^{*} \phi+g\left(\phi^{*} \phi\right)^{2}
$$

where $\nabla_{\mu}$ represents the ordinary spacetime covariant derivative, and a metric with signature $(+,-,-,-)$ is used. The coupling constants $\lambda, f$, and $g$, as well as the $\phi$ particle mass $m$, are assumed to be positive, real quantities. The stable vacuum state of the theory is located by $|\chi|=\eta, \phi=0$, and the $\chi$ particle mass is $m_{\chi}=(2 \lambda)^{\frac{1}{2}} \eta$.

The general field equations which follow from (1) are given by

$$
\begin{gathered}
\square \chi+\left[2 \lambda\left(\chi^{*} \chi-\frac{1}{2} \eta^{2}\right)+2 f \phi^{*} \phi\right] \chi=0, \\
\square \phi-e^{2} \phi A^{\mu} A_{\mu}+i e\left(2 A^{\mu} \partial_{\mu} \phi+\phi \nabla_{\mu} A^{\mu}\right) \\
+\left[2 f\left(\chi^{*} \chi-\frac{1}{2} \eta^{2}\right)+m^{2}+2 g \phi^{*} \phi\right] \phi=0, \\
\begin{aligned}
\nabla_{\mu} F^{\mu \nu} & =\square A^{\nu}=e J_{T}^{\nu} \\
& =i e\left[\phi^{*} \partial^{\nu} \phi-\phi \partial^{\nu} \phi^{*}\right]-2 e^{2} \phi^{*} \phi A^{\nu},
\end{aligned}
\end{gathered}
$$

where $\square \equiv \nabla_{\mu} \nabla^{\mu}$ and we have chosen the Lorentz gauge $\nabla_{\mu} A^{\mu}=0$, and the conserved total current density is

$$
\begin{aligned}
J_{T}^{\nu} & =i\left[\phi^{*}\left(D^{\nu} \phi\right)-\phi\left(D^{\nu} \phi\right)^{*}\right] \\
& =i\left[\phi^{*} \partial^{\nu} \phi-\phi \partial^{\nu} \phi^{*}\right]-2 e \phi^{*} \phi A^{\nu}, \quad \nabla_{\nu} J_{T}^{\nu}=0 .
\end{aligned}
$$

\section{B. Parametrization and Primary Fields}

Let us consider a parametrization of the various fields as given by

$$
\chi=\frac{1}{\sqrt{2}}[R(r)+G(\vec{r}, t)] e^{i \theta}
$$




$$
\begin{gathered}
\phi=\frac{1}{\sqrt{2}}[F(r)+H(\vec{r}, t)] e^{i \psi(t, z)}, \\
A_{\mu}=B_{\mu}+a_{\mu}(\vec{r}, t), \quad B_{\mu} \equiv \frac{1}{e}[P(r)-1] \partial_{\mu} \psi(t, z), \\
F_{\mu \nu}=B_{\mu \nu}+f_{\mu \nu}, \quad B_{\mu \nu} \equiv \partial_{\mu} B_{\nu}-\partial_{\nu} B_{\mu}, \quad f_{\mu \nu} \equiv \partial_{\mu} a_{\nu}-\partial_{\nu} a_{\mu} .
\end{gathered}
$$

The total conserved current $J_{T}^{\nu}$ can be written as

$$
J_{T}^{\nu}=J^{\nu}+j^{\nu}
$$

where $J^{\nu}$ is obtained from (可) upon setting $G=0, H=0$, and $a_{\mu}=0$, so that in this case one obtains

$$
\begin{array}{ll}
\chi=\frac{1}{\sqrt{2}} R(r) e^{i \theta}, & \phi=\frac{1}{\sqrt{2}} F(r) e^{i \psi(t, z)} \\
A_{\mu}=B_{\mu}=\frac{1}{e}[P(r)-1] \partial_{\mu} \psi, & J^{\nu}=-F^{2} P \partial^{\nu} \psi
\end{array}
$$

The fields in (13) will be referred to as the primary fields, and the fields

$$
\chi_{1} \equiv \frac{1}{\sqrt{2}} G(\vec{r}, t) e^{i \theta}, \quad \phi_{1} \equiv \frac{1}{\sqrt{2}} H(\vec{r}, t) e^{i \psi(t, z)}, \quad a_{\mu}=A_{\mu}-B_{\mu}
$$

will be referred to as the excitation fields.

A basic ansatz for the superconducting global string can be built from the primary fields, so that upon setting the excitation fields equal to zero, the primary field equations which follow from (4) - (6) can be written as

$$
\begin{gathered}
\frac{1}{r} \partial_{r}\left(r \partial_{r} R\right)-\frac{R}{r^{2}}-\left[\lambda\left(R^{2}-\eta^{2}\right)+f F^{2}\right] R=0, \\
\frac{1}{r} \partial_{r}\left(r \partial_{r} F\right)+F P^{2} \partial_{\mu} \psi \partial^{\mu} \psi-\left[f\left(R^{2}-\eta^{2}\right)+m^{2}+g F^{2}\right] F=0 \\
\frac{1}{r} \partial_{r}\left(r \partial_{r} P\right)-e^{2} F^{2} P=0 .
\end{gathered}
$$

The potential in (3), in terms of the primary scalar fields, becomes

$$
V=\frac{1}{4} \lambda\left(R^{2}-\eta^{2}\right)^{2}+\frac{1}{2} f\left(R^{2}-\eta^{2}\right) F^{2}+\frac{1}{2} m^{2} F^{2}+\frac{1}{4} g F^{4} .
$$

Inside the string, $R \rightarrow 0$ as $r \rightarrow 0$, so that in the string core the potential $V$ is minimized by a value of $F$ given by 


$$
F_{0}=\left[\frac{\left(f \eta^{2}-m^{2}\right)}{g}\right]^{\frac{1}{2}},
$$

whereas in the true vacuum region, as $r \rightarrow \infty$, and $R \rightarrow \eta$, the potential $V$ is minimized by a value of $F$ given by $F=0$. Therefore, we take the boundary conditions for the primary field equations (15-17) describing the superconducting global string to be

$$
\begin{gathered}
R \rightarrow 0, \quad F \rightarrow F_{0}, \quad P \rightarrow 1, \quad \partial_{r} P \rightarrow 0, \quad \text { as } r \rightarrow 0, \\
R \rightarrow \eta, \quad F \rightarrow 0, \quad B_{\mu \nu} \rightarrow 0, \quad \text { as } r \rightarrow \infty .
\end{gathered}
$$

It is furthermore assumed that the quantity $\partial_{\mu} \psi \partial^{\mu} \psi$ in (16) is given by

$$
\partial_{\mu} \psi \partial^{\mu} \psi=K
$$

where $K$ is a constant, and the radius of the string is taken to be $r_{0} \approx m_{\chi}^{-1}=$ $\left[(2 \lambda)^{\frac{1}{2}} \eta\right]^{-1}$.

\section{Charge, Current, and Electromagnetic Fields}

For the basic superconducting global string ansatz described by (13) with $j^{\nu}=0$, the primary current density $J^{\nu}=-F^{2}(r) P(r) \partial^{\nu} \psi(t, z)$ is conserved so that

$$
\nabla_{\mu} J^{\mu}=0 \Rightarrow\left(\partial_{0}^{2}-\partial_{z}^{2}\right) \psi(t, z) \equiv \square_{2} \psi(t, z)=0,
$$

where $\square_{2} \equiv\left(\partial_{0}^{2}-\partial_{z}^{2}\right)$. The linear charge density and the current that are enclosed within a radius $r$ are

$$
\begin{gathered}
Q_{e n}(r)=2 \pi e \int_{0}^{r} J^{0} r^{\prime} d r^{\prime}=-2 \pi e J(r) \partial_{0} \psi, \\
I_{e n}(r)=2 \pi e \int_{0}^{r} J^{z} r^{\prime} d r^{\prime}=2 \pi e J(r) \partial_{z} \psi,
\end{gathered}
$$

respectively, with $J(r) \equiv \int_{0}^{r} F^{2} P r^{\prime} d r^{\prime}$. The total (primary) charge per unit length and the total (primary) current (due to the primary current density $J^{\mu}$ ) carried by the string are $Q=Q_{e n}(\infty) \approx Q_{e n}\left(r_{0}\right)$ and $I=I_{e n}(\infty) \approx I_{e n}\left(r_{0}\right)$, respectively, where $r_{0} \sim m_{\chi}^{-1}=(\sqrt{2 \lambda} \eta)^{-1}$ is the string core radius. The electric and magnetic fields that are generated by the primary current density are therefore given by

$$
E_{r}(r)=\frac{Q_{e n}(r)}{2 \pi r}, \quad B_{\theta}(r)=\frac{I_{e n}(r)}{2 \pi r} .
$$


By (23) the general solution for $\psi$ can be expressed in terms of travelling waves,

$$
\psi(t, z)=\psi_{+}(t+z)+\psi_{-}(t-z)
$$

In terms of the light cone coordinates $\xi_{ \pm} \equiv t \pm z$, we have $\psi=\psi_{+}\left(\xi_{+}\right)+\psi_{-}\left(\xi_{-}\right)$. However, when the constraint given by (22) is taken into account, we have for the general solutions $\psi=\omega t+k z+$ const with $\omega^{2}-k^{2}=K$ for the case $K \neq 0$, whereas for the case $K=0$,

$$
\psi=\psi(\xi), \quad \text { where either } \xi=\xi_{+} \quad \text { or } \xi=\xi_{-}, \quad(K=0) .
$$

Let us further require that the current density $J_{\mu}$, and therefore $\partial_{\mu} \psi$, be a bounded function of $\xi$, so that for the case $K=0, \psi$ can include the description of a pulse with arbitrary shape travelling either up the string in the $+z$ direction or down the string in the $-z$ direction, in addition to a static charge and current. (Note that, for $K=0, Q_{e n}, I_{e n}, E_{r}$, and $B_{\theta}$ can be considered as functions of $r, t$, and $z$, in general.)

\section{EXCITATION FIELDS}

\section{A. Linearized Excitation Field Equations}

Now consider the case where the excitation fields described by (14) are nonvanishing, but are generally considered to be sufficiently small in comparison to the primary fields. Using the parametrized fields of (8) -(11) the field equations given by (4) (匹) yield the linearized excitation field equations

$$
\begin{gathered}
\square G+\frac{G}{r^{2}}+2 i \partial_{\mu} G \partial^{\mu} \theta+\lambda G\left(3 R^{2}-\eta^{2}\right)+f F(F G+2 R H)=0, \\
\square H-K H+i\left(2 \partial_{\mu} H \partial^{\mu} \psi+H \square \psi\right)-e^{2} B_{\mu}\left(B^{\mu} H+2 a^{\mu} F\right) \\
-2 e\left[B^{\mu}\left(H \partial_{\mu} \psi-i \partial_{\mu} H\right)+a^{\mu}\left(F \partial_{\mu} \psi-i \partial_{\mu} F\right)\right] \\
+f\left[\left(R^{2}-\eta^{2}\right) H+2 F R G\right]+\left(m^{2}+3 g F^{2}\right) H=0 \\
\nabla_{\mu} f^{\mu \nu}=\square a^{\nu}=e j^{\nu}=-e F\left[2 H\left(\partial^{\nu} \psi+e B^{\nu}\right)+e F a^{\nu}\right]
\end{gathered}
$$

and $\nabla_{\mu} j^{\mu}=0$ implies that

$$
\partial_{\nu}(F H) \partial^{\nu} \psi+F H \square \psi+e \nabla_{\nu}\left(F H B^{\nu}\right)+F \partial_{\nu} F a^{\nu}=0 .
$$




\section{B. Approximations and Ansatz}

Equations (29) - (32) can be simplified considerably by making some simple assumptions and implementing an ansatz. Let it first be assumed that solutions exist for which there is a negligible back reaction upon the global string field, i.e. let us make the approximation $G \approx 0$. This is seen to be an approximate solution of (29) provided that $f F R H \approx 0$, which is satisfied both inside the string core $(R \rightarrow 0)$ and outside the string $(F H \rightarrow 0)$. It is also convenient to regard the global string as a tube of false vacuum with a radius $r_{0} \approx\left[(2 \lambda)^{\frac{1}{2}} \eta\right]^{-1}$, and make the approximations

$$
\left.F \approx\left\{\begin{array}{c}
F_{0} \\
0
\end{array}\right\}, \quad R \approx\left\{\begin{array}{l}
0 \\
\eta
\end{array}\right\}, \quad P \approx\left\{\begin{array}{l}
1 \\
0
\end{array}\right\}, \quad \text { for } \quad \begin{array}{l}
r \leq r_{0} \\
r>r_{0}
\end{array}\right\} .
$$

Along with these approximations, let us impose the ansatz conditions

$$
\partial_{\mu} \psi \partial^{\mu} \psi=K=0, \quad a^{\mu} \partial_{\mu} F=0, \quad a^{\mu} \partial_{\mu} \psi=0
$$

The first condition in (34) allows travelling waves of charge and current to propagate either up the string or down the string, since $\psi=\psi(\xi)$, where either $\xi=\xi_{+}$or $\xi=\xi_{-}$, and the second condition, which is a simplifying condition, implies that $a^{r}=0$ for the general case where $F=F(r)$ or $\partial_{\mu} F \neq 0$. The last condition of (34), along with (32) and $a^{\mu} \partial_{\mu} F=0$, implies, for the general case where $P \neq 0$, i.e. $e B_{\mu} \neq-\partial_{\mu} \psi$, the auxiliary condition

$$
\partial_{\mu} H \partial^{\mu} \psi=0
$$

\section{Approximate Excitation Field Equations}

Using the fact that $\square \psi=0$, the approximation $G \approx 0$, along with (33) - (35), the linearized equations reduce greatly. Inside the string core, for $r \leq r_{0}$, we have

$$
\begin{gathered}
\square H+M^{2} H=0, \quad M \equiv(2 g)^{\frac{1}{2}} F_{0}, \\
\nabla_{\mu} f^{\mu \nu}=\square a^{\nu}=e j^{\nu}=-e F_{0}\left(2 H \partial^{\nu} \psi+e F_{0} a^{\nu}\right),
\end{gathered}
$$

where $\nabla_{\mu} j^{\mu} \equiv 0$, and outside the string core, for $r>r_{0}$,

$$
\begin{gathered}
\square H+m^{2} H=0, \\
\nabla_{\mu} f^{\mu \nu}=\square a^{\nu}=e j^{\nu}=0 .
\end{gathered}
$$


In addition, $H$ must satisfy the auxiliary condition $\partial_{\mu} H \partial^{\mu} \psi=0$, given by (35). Note that the field $H(\vec{r}, t)$ thus has the appearance of a massive Higgs field, with mass $M=(2 g)^{\frac{1}{2}} F_{0}$ inside the string and mass $m$ outside the string. However, solutions shall be found for which the field $H$ behaves as an effectively massless field that can propagate along the string at the speed of light, with the mass parameters serving to modulate the radial profile of the field. This type of behavior will be seen to arise in response to the constraint $\partial_{\mu} H \partial^{\mu} \psi=0$ that has been imposed. Similarly, the photon excitation field $a^{\mu}$ has the appearance of a massive vector field inside the string core and a massless vector field outside the string, but the solutions to be found will suggest that the vector field $a^{\mu}$ can actually behave as an effectively massless field propagating along the string at the speed of light along with the scalar field $H$.

\section{SOLUTIONS}

We regard $H(\vec{r}, t)$ as being small compared to $F(r)$, i.e. $|H| \ll F(r)$. In addition, the constraint $\partial_{\mu} H \partial^{\mu} \psi=0$ must be satisfied. It follows from the boundary conditions given by (20) and (21) that $H$ and $a_{\mu}$ must satisfy the boundary conditions

$$
\begin{aligned}
|H| & \ll F_{0}, \quad a_{\mu} \rightarrow 0, \quad \text { as } \quad r \rightarrow 0, \\
H & \rightarrow 0, \quad\left|a_{\mu}\right| \ll\left|B_{\mu}\right|, \quad \text { as } r \rightarrow \infty .
\end{aligned}
$$

\section{A. Scalar Field}

Let us write $H(\vec{r}, t)$ in the form $H=H(r, \theta, \xi)$. Then from $\partial_{\mu} \psi \partial^{\mu} \psi=0$ it follows that $\psi=\psi(\xi)$, as given by (28). From the condition given by (35), $\partial_{\mu} H \partial^{\mu} \psi=$ $0 \Rightarrow \partial_{+} H \partial_{-} \psi+\partial_{-} H \partial_{+} \psi=0$, where $\partial_{ \pm} \equiv \partial / \partial \xi_{ \pm}$. Therefore, if $\psi=\psi\left(\xi_{+}\right)$, then $\partial_{-} \psi=0$, which implies that $\partial_{-} H=0$, and therefore $H=H\left(\xi_{+}\right)$. On the other hand, if $\psi=\psi\left(\xi_{-}\right)$, then $\partial_{+} \psi=0$, so that $\partial_{+} H=0$, and therefore $H=H\left(\xi_{-}\right)$. In either case, if $\psi=\psi(\xi)$, then $H=H(\xi)$, where either $\xi=\xi_{+}$or $\xi=\xi_{-}$, and consequently

$$
\square_{2} H(r, \theta, \xi) \equiv\left(\partial_{0}^{2}-\partial_{z}^{2}\right) H(r, \theta, \xi)=4 \partial_{+} \partial_{-} H(r, \theta, \xi)=0 \text {. }
$$

Therefore, in general, both $\psi$ and $H$ can describe pulses that propagate along together at the speed of light either up the string or down the string.

Writing $H_{l}(r, \theta, \xi)=h_{l}(\xi) q_{l}(r) \sin (l \theta)$, where $l=0,1,2, \cdots$, (36) reduces, for $r \leq r_{0}$, to

$$
\partial_{r}^{2} q_{l}+\frac{1}{r} \partial_{r} q_{l}-\left(M^{2}+\frac{l^{2}}{r^{2}}\right) q_{l}=0,
$$

which is solved by $q_{l}=\alpha_{l} I_{l}(M r)$, where $I_{l}$ is a hyperbolic Bessel function of order $l$, and is zero (for $l \neq 0$ ) at $r=0$, and $\alpha_{l}$ is a constant. (Note that $H_{0}(r, \theta, \xi)=0$.) The solution $H_{l}$ can then be displayed as 


$$
H_{l}(r, \theta, \xi)=\alpha_{l} h_{l}(\xi) I_{l}(M r) \sin (l \theta), \quad\left(r \leq r_{0}\right)
$$

where $h(\xi)$ is a sufficiently bounded, but otherwise arbitrary, function of $\xi$. The general solution for $H$ inside the string core is given by a linear superposition, $H(r, \theta, \xi)=\sum_{l} H_{l}(r, \theta, \xi)$.

Outside the string core, for $r>r_{0}$, a similar type of solution exists, except that $M^{2}$ must be replaced by $m^{2}$, and the allowed radial solutions that conform to the boundary conditions are the hyperbolic Bessel functions $K_{l}(m r)$, which are finite, rapidly decreasing functions of $r$ outside the string core. The exterior solutions are therefore given by

$$
H_{l}(r, \theta, \xi)=\beta_{l} h_{l}(\xi) K_{l}(m r) \sin (l \theta), \quad\left(r>r_{0}\right) .
$$

The continuity of $H(r, \theta, \xi)$ demands that the interior and exterior solutions be joined at $r=r_{0}$, and since $I_{l}(M r)$ is an increasing function of $r$, while $K_{l}(m r)$ is a decreasing function of $r$, we conclude that, within the context of the approximations employed, the scalar excitation field $H(r, \theta, \xi)$ is concentrated at the surface of the string, i.e. at $r=r_{0}$, and travels in a nondispersive way along the string at the speed of light together with the primary charge/current pulse arising from the current density $J_{\mu}$. The profile of the scalar field along the string, given by $h_{l}(\xi)$, can be a function of arbitrary shape, subject to the requirement that $H(r, \theta, \xi)$ be small compared to the primary field $F(r)$.

\section{B. Vector Field}

In addition to the boundary conditions given by (40), the vector field $a^{\mu}(\vec{r}, t)$ must be further restricted by requiring that the electric field $\vec{e}$ and the magnetic field $\vec{b}$ that are generated from $a^{\mu}$ be well defined at $r=0$. In particular, we require

$$
e_{r} \rightarrow 0, \quad e_{\theta} \rightarrow 0, \quad b_{r} \rightarrow 0, \quad b_{\theta} \rightarrow 0, \quad \text { as } r \rightarrow 0
$$

For the interior region of the string, $r \leq r_{0}$, equations (34), (35), and (37) are seen to be solved by

$$
a_{l}^{\mu}(r, \theta, \xi)=\gamma H_{l}(r, \theta, \xi) \partial^{\mu} \psi=\gamma \alpha_{l} h_{l}(\xi) I_{l}(M r) \sin (l \theta) \partial^{\mu} \psi, \quad\left(r \leq r_{0}\right)
$$

where $H_{l}$ is the interior scalar field solution given by (43), provided that the constant $\gamma$ is given by

$$
\gamma=\frac{2 e F_{0}}{\left(M^{2}-e^{2} F_{0}^{2}\right)}=\frac{2 e}{\left(2 g-e^{2}\right) F_{0}} .
$$

Note that for $l=0, a_{0}^{\mu}=0$. For this solution to satisfy the conditions given by (45) we must require that $\alpha_{1}=0$, which implies that $\beta_{1}=0$, due to continuity of the scalar field $H$ at $r=r_{0}$. We therefore have 


$$
\alpha_{1}=\beta_{1}=0, H_{0}(r, \theta, \xi)=H_{1}(r, \theta, \xi)=0, a_{0}^{\mu}(r, \theta, \xi)=a_{1}^{\mu}(r, \theta, \xi)=0 .
$$

Outside the string, for $r>r_{0}$, let $a^{\mu}$ be displayed as $a_{l}^{\mu}(r, \theta, \xi)=$ $c_{l} h_{l}(\xi) f_{l}(r) \sin (l \theta) \partial^{\mu} \psi$, where $c_{l}$ is a constant. Then (39) reduces to

$$
\partial_{r}^{2} f_{l}+\frac{1}{r} \partial_{r} f_{l}-\frac{l^{2}}{r^{2}} f_{l}=0
$$

which is solved by $f_{l}(r)=r^{-l}(l \neq 0)$. Upon requiring continuity of the interior and exterior solutions at $r=r_{0}$, we must have $c_{0}=c_{1}=0$ so that $a_{0}^{\mu}=a_{1}^{\mu}=0$ for all $r$. The exterior solution for the vector field is then given by

$$
\begin{aligned}
a_{l}^{\mu}(r, \theta, \xi) & =c_{l} h_{l}(\xi) f_{l}(r) \sin (l \theta) \partial^{\mu} \psi \quad, \quad\left(r>r_{0}\right) \\
& =c_{l} h_{l}(\xi) r^{-l} \sin (l \theta) \partial^{\mu} \psi
\end{aligned}
$$

Since the vector field increases radially for $r \leq r_{0}$ and then decreases radially for $r>r_{0}, a^{\mu}$ is seen to be concentrated at $r=r_{0}$, with a tail that falls off as $r^{-l}$ outside the string.

\section{Electromagnetic Fields}

The electromagnetic fields exterior to the string can be obtained from (50), and are given by

$$
\begin{gathered}
e_{r, l}=-\partial_{r} a_{l}^{0}=l c_{l} h_{l}(\xi) r^{-(l+1)} \sin (l \theta) \partial^{0} \psi=\frac{l}{r} a_{l}^{0}, \\
e_{\theta, l}=-\frac{1}{r} \partial_{\theta} a_{l}^{0}=-l c_{l} h_{l}(\xi) r^{-(l+1)} \cos (l \theta) \partial^{0} \psi=-\frac{l}{r} \cot (l \theta) a_{l}^{0}, \\
b_{r, l}=\frac{1}{r} \partial_{\theta} a_{l}^{z}=l c_{l} h_{l}(\xi) r^{-(l+1)} \cos (l \theta) \partial^{z} \psi=\frac{l}{r} \cot (l \theta) a_{l}^{z}, \\
b_{\theta, l}=-\partial_{r} a_{l}^{z}=l c_{l} h_{l}(\xi) r^{-(l+1)} \sin (l \theta) \partial^{z} \psi=\frac{l}{r} a_{l}^{z} .
\end{gathered}
$$

The Poynting vector associated with these fields is

$$
\begin{aligned}
\vec{s}_{l}=\vec{e}_{l} \times \vec{b}_{l} & =\hat{z} l^{2} c_{l}^{2} h_{l}^{2}(\xi) r^{-2(l+1)} \partial^{0} \psi \partial^{z} \psi=\hat{z} \frac{l^{2}}{r^{2}} a_{l}^{0} a_{l}^{z} \\
& =\mp \hat{z}\left(l c_{l} h_{l}(\xi) r^{-(l+1)}\right)^{2}\left(\partial_{ \pm} \psi\right)^{2}
\end{aligned}
$$

which indicates that the electromagnetic power flows along the string with the excitation pulse. 
For a superconducting string formed during a Grand Unified Theory (GUT) symmetry breaking phase transition, with a GUT mass scale characterized by $\eta$, the linear charge density $Q$ and the current $I$ can be enormous [1], with $I_{\max } \sim O(e \eta)$. Let us consider, for example, a scalar field phase function $\psi(\xi)$, where we take $\xi=\xi_{-}=(t-z)$, for definiteness. Then $\partial_{0} \psi=\partial_{-} \psi=\partial_{\xi} \psi$, and $\partial_{z} \psi=-\partial_{\xi} \psi$, and by (24) and (25) the primary linear charge density and current are given by $Q=I \approx-2 \pi e J\left(r_{0}\right) \partial_{\xi} \psi$, where $J\left(r_{0}\right) \sim \frac{F_{0}^{2} r_{0}^{2}}{2} \sim \frac{F_{0}^{2}}{4 \lambda \eta^{2}}$, so that $I \sim-\left(\frac{e \pi F_{0}^{2}}{2 \lambda \eta^{2}}\right) \partial_{\xi} \psi$, which implies that

$$
\partial_{\xi} \psi \sim-\frac{I}{e \pi F_{0}^{2} r_{0}^{2}} \sim-I\left(\frac{2 \lambda \eta^{2}}{e \pi F_{0}^{2}}\right) .
$$

By (51) - (54) it then follows that

$$
\left|\vec{e}_{l}\right|=\left|\vec{b}_{l}\right|=l r^{-(l+1)}\left|c_{l} h_{l}(\xi) \partial_{\xi} \psi\right| \sim \frac{l}{r^{(l+1)}}\left|c_{l} h_{l}(\xi)\right|\left(\frac{2 \lambda \eta^{2}}{e \pi F_{0}^{2}}\right) I .
$$

As a specific example, consider the phase function $\psi=\omega \xi+\psi_{p}(\xi)$, where $\xi=t-z$ and $\psi_{p}$ is a bounded function of $\xi$ describing a pulse travelling along the string. By defining the constant current $I_{0} \equiv-\omega\left(\frac{e \pi F_{0}^{2}}{2 \lambda \eta^{2}}\right)$ and the pulse current $I_{p}(\xi) \equiv-\left(\frac{e \pi F_{0}^{2}}{2 \lambda \eta^{2}}\right) \partial_{\xi} \psi_{p}(\xi)$, (57) gives field strengths $\left|\vec{e}_{l}\right|=\left|\vec{b}_{l}\right| \sim l r^{-(l+1)}\left(\frac{2 \lambda \eta^{2}}{e \pi F_{0}^{2}}\right)\left|c_{l} h_{l}(\xi)\left(I_{0}+I_{p}(\xi)\right)\right|$, with $h_{l}(\xi)$ being a bounded function of $\xi$ (so that $H(\vec{r}, t)$ cannot grow too large in comparison to $F(r))$ satisfying $\partial_{\mu} h_{l} \partial^{\mu} \psi=0$. Because the current $I$ can be so large, the fields $\vec{e}$ and $\vec{b}$, although perhaps quite small in comparison to the primary fields $E_{r}=\frac{Q}{2 \pi r}$ and $B_{\theta}=\frac{I}{2 \pi r}$, can themselves be quite large. It is furthermore interesting to note that while the primary electric field $\vec{E}$ has only a nonvanishing radial component and the primary magnetic field $\vec{B}$ has only a nonvanishing angular component, the fields $\vec{e}$ and $\vec{b}$ each have nonvanishing radial and angular components, in general.

\section{SUMMARY AND DISCUSSION}

A model of a superconducting global string has been examined where the spontaneous breaking of a $U(1)$ global symmetry, giving rise to the global string, is accompanied by the formation of a charged scalar condensate (coupled to a $U(1)$ gauge field) in the core of the string. The string supports a linear charge density $Q$ and carries a current $I$. Attention has been focused upon the special case for which, by (24) and (25), $Q^{2}-I^{2}=(2 \pi e J(r))^{2} \partial_{\mu} \psi \partial^{\mu} \psi=0$, which is a Lorentz invariant condition. This type of condition is invoked, for example, for certain chiral vorton models [6], [7], and has the feature that, by (26), the electric and magnetic fields have equal magnitudes, i.e. $|\vec{E}|=|\vec{B}|$, so that particle pair production due to a strong electric field near the string is suppressed. A basic ansatz which describes a straight superconducting string has been examined. For this basic ansatz, the modulus of the 
scalar condensate field, $|\phi|$, and the gauge field structure function $P$, are considered to be functions only of the radial distance $r$ from the center of the string core, and the phase $\psi$ of the scalar condensate depends only upon the coordinate $z$ along the string and the time $t$. Fluctuations of the scalar and vector fields about the primary fields of the basic ansatz have been considered, and linear field equations for these fluctuation, or excitation, fields have been obtained.

The behavior of the excitation fields is determined by a set of nontrivial coupled equations, and approximate solutions have been obtained by making certain approximations and imposing simplifying conditions, such as $Q^{2}-I^{2}=0\left(\partial_{\mu} \psi \partial^{\mu} \psi=0\right)$, along with others. These solutions are particular solutions, which can be associated with possible nonradiative excitation modes for the scalar and vector fields of the model, and are expected to be special cases of more general solutions, which may include descriptions of radiative excitation modes. The equation of motion for the scalar excitation field $H$ appears to describe a massive 'Higgs' mode, but, because of an auxiliary condition which has been imposed, the particular solutions obtained describe an effectively massless scalar mode which propagates along the string with the primary current pulse at the speed of light. Similarly, the equation of motion for the vector field $a^{\mu}$ appears to describe a massive photon, but the particular solutions obtained describe an effectively massless vector field which propagates along the string with the scalar field. In each case, the effect of the mass parameter is to modulate the radial profile of the effectively massless mode. The nonvanishing solutions for $H_{l}$ and $a_{l}^{\mu}$ have nonzero angular momentum contributions, i.e. $l \neq 0,1$.

Both the scalar and photon excitation fields contribute to the current density $j^{\mu}$ and thus serve as sources for electromagnetic fields. The current density $j^{\mu}$ generates an electric field with components $e_{r}$ and $e_{\theta}$, and a magnetic field with components $b_{r}$ and $b_{\theta}$. Furthermore, since $j_{l}^{\mu}$ vanishes for $l=0$, the total charge per unit length $q$ and the total current $i$ generated by $j^{\mu}$ vanish, with $q=i=0$. The Poynting vector $\vec{s}$ resulting from $\vec{e}$ and $\vec{b}$ possesses a nonvanishing component only in a direction parallel to the string, so that $s_{r}=0$, indicating that the string does not absorb or emit electromagnetic radiation. The particular solutions obtained therefore describe nonradiative excitation modes.

It can be noted that $H(\xi)$ and $a^{\mu}(\xi)$ are somewhat arbitrary functions of $\xi$ (subject to the requirement that they be sufficiently small bounded functions of $\xi$ ), with their profiles along the string presumably being dictated by initial conditions, which, in turn, are produced by interactions of the superconducting string with other strings, charged particles, or electromagnetic fields. Therefore, if the superconducting string experiences any electromagnetic interactions, excitation fields are expected to be produced. It is hoped that the special class of excitation field solutions studied here can yield some insight regarding more general phenomena involving superconducting cosmic strings. 
[1] E. Witten, Nucl. Phys. B 249, 557 (1985)

[2] Ö. F. Dayi, H. J. W. Müller-Kirsten, A. V. Shurgaia, and D. H. Tchrakian, Phys. Lett. B 286, 234 (1992)

[3] P. Peter, Phys. Rev. D 45, 1091 (1992)

[4] A. Vilenkin, Phys. Rep. 121, 263 (1985)

[5] A. Vilenkin and A. E. Everett, Phys. Rev. Lett. 48, 1867 (1982)

[6] R. L. Davis, in The Formation and Evolution of Cosmic Strings, edited by G. Gibbons, S. Hawking, and T. Vachaspati (Cambridge University Press, Cambridge, 1990)

[7] J. R. Morris, Nuovo Cimento A 106, 355 (1993) 